\title{
PROTO-EXACT CATEGORIES OF MATROIDS, HALL ALGEBRAS, AND K-THEORY
}

\author{
CHRISTOPHER EPPOLITO, JAIUNG JUN, AND MATT SZCZESNY
}

\begin{abstract}
This paper examines the category Mat. of pointed matroids and strong maps from the point of view of Hall algebras. We show that Mat. has the structure of a finitary proto-exact category - a non-additive generalization of exact category due to Dyckerhoff-Kapranov. We define the algebraic K-theory $K_{*}$ (Mat.) of Mat. via the Waldhausen construction, and show that it is non-trivial, by exhibiting injections

$$
\pi_{n}^{\text {S }}(\mathrm{S}) \hookrightarrow K_{n}\left(\text { Mat. }_{\bullet}\right)
$$

from the stable homotopy groups of spheres for all $n$. Finally, we show that the Hall algebra of Mat. is a Hopf algebra dual to Schmitt's matroidminor Hopf algebra.
\end{abstract}

\section{INTRODUCTION}

In this paper we examine the category of pointed matroids and strong maps from the perspective of Hall algebras, which have traditionally been studied in representation theory. This perspective sheds new light on certain combinatorial Hopf algebras built from matroids, and opens the door to defining algebraic K-theory of matroids. The rest of this introduction is devoted to introducing the main actors.

1.1 Hall algebras of Abelian and exact categories. The study of Hall algebras is by now a well-established area with several applications in representation theory and algebraic geometry (see [21] for a very nice overview). We briefly recall the generic features of the most basic version of this construction. Given an abelian category $\mathcal{C}$, let

$$
\mathbf{F L}_{i}(\mathcal{C}):=\left\{A_{0} \subseteq A_{1} \subseteq \cdots \subseteq A_{i} \mid A_{k} \in \mathrm{Ob}(\mathcal{C})\right\}
$$

denote the stack parametrizing isomorphism classes of flags of objects in $\mathcal{C}$ of length $i+1$ (viewed here simply as a set). Thus

$$
\mathbf{F L}_{0}(\mathcal{C})=\operatorname{Iso}(\mathcal{C})
$$

2010 Mathematics Subject Classification. 18D99(primary), 05B35, 16T30, 19A99, 19D99 (secondary).

Key words and phrases. matroid, matroid strong maps, matroid-minor Hopf algebra, Hall algebra, Protoexact category, K-theory. 
the moduli stack of isomorphism classes of objects of $\mathcal{C}$, and

$$
\mathbf{F L}_{1}(\mathcal{C})=\left\{A_{0} \subseteq A_{1} \mid A_{0}, A_{1} \in \mathrm{Ob}(\mathcal{C})\right\}
$$

is the usual Hecke correspondence. We have maps

$$
\pi_{i}: \mathrm{FL}_{1}(\mathcal{C}) \rightarrow \operatorname{Iso}(\mathcal{C}), i=1,2,3,
$$

where

$$
\begin{aligned}
& \pi_{1}\left(A_{0} \subseteq A_{1}\right)=A_{0}, \\
& \pi_{2}\left(A_{0} \subseteq A_{1}\right)=A_{1}, \\
& \pi_{3}\left(A_{0} \subseteq A_{1}\right)=A_{1} / A_{0} .
\end{aligned}
$$

We may then attempt to define the Hall algebra of $\mathcal{C}$ as the space of $\mathbb{Q}$-valued functions on $\operatorname{Iso}(\mathcal{C})$ with finite support, i.e.

$$
\mathrm{H}_{\mathcal{C}}=\mathbb{Q}_{c}[\operatorname{Iso}(\mathcal{C})]
$$

with the convolution product defined for $f, g \in \mathrm{H}_{\mathcal{C}}$

$$
f \star g:=\pi_{2 *}\left(\pi_{3}^{*}(f) \pi_{1}^{*}(g)\right),
$$

where $\pi_{i}^{*}$ denotes the usual pullback of functions and $\pi_{i *}$ denotes integration along the fiber. To make this work, one has to impose certain finiteness conditions on $\mathcal{C}$. The simplest, and most restrictive such condition is that $\mathcal{C}$ is finitary, which means that $\operatorname{Hom}\left(M, M^{\prime}\right)$ and $\operatorname{Ext}^{1}\left(M, M^{\prime}\right)$ are finite sets for any pair of objects $M, M^{\prime} \in \mathcal{C}$.

$\mathrm{H}_{\mathcal{C}}$ is spanned by $\delta$-functions $\delta_{[M]},[M] \in \operatorname{Iso}(\mathcal{C})$ supported on individual isomorphism classes, and the product (2) can be explicitly written

$$
\delta_{[M]} \star \delta_{[N]}=\sum_{R \in \operatorname{Iso}(\mathcal{C})} \mathbf{P}_{M, N}^{R} \delta_{R}
$$

where

$$
\mathbf{P}_{M, N}^{R}:=\#|\{L \subseteq R, L \simeq N, R / L \simeq M\}|
$$

The number

$$
\mathbf{P}_{M, N}^{R}|\operatorname{Aut}(M)||\operatorname{Aut}(N)|
$$

counts the isomorphism classes of short exact sequences of the form

$$
0 \rightarrow N \rightarrow R \rightarrow M \rightarrow 0,
$$

where $\operatorname{Aut}(M)$ is the automorphism group of $M$. Thus, product in $\mathrm{H}_{\mathcal{C}}$ encodes the structure of extensions in $\mathcal{C}$.

Important examples of finitary categories are $\mathcal{C}=\operatorname{Rep}\left(Q, \mathbb{F}_{q}\right)$ - the category of representations of a quiver $Q$ over a finite field $\mathbb{F}_{q}$, and $\mathcal{C}=\operatorname{Coh}\left(X / \mathbb{F}_{q}\right)$ - the category of 
coherent sheaves on a smooth projective variety $X$ over $\mathbb{F}_{q}$. In these examples, the structure constants of $\mathrm{H}_{\mathcal{C}}$ depend on the parameter $q$, and $\mathrm{H}_{\mathcal{C}}$ recovers (parts of) quantum groups and their generalizations.

The basic recipe above extends more generally to the case where $\mathcal{C}$ is a finitary Quillen exact category (see [13]). Exact categories can be viewed as a strictly full extension-closed subcategories of Abelian categories, and can be equivalently described in terms of classes $(\mathfrak{M}, \mathfrak{E})$ of admissible mono/epi-morphisms. For example, the category of vector bundles (i.e. locally free sheaves) on a smooth projective curve $X / \mathbb{F}_{q}$ is an exact category, being an extension-closed full subcategory of $\operatorname{Coh}\left(X / \mathbb{F}_{q}\right)$, with $(\mathfrak{M}, \mathfrak{E})$ consisting of those monos/epis which are locally split. It is not Abelian, since the kernel/cokernel of a morphism of locally free sheaves may be a coherent sheaf that is not locally free. In this example, the stack Iso $(\mathcal{C})$ is the domain of definition of automorphic forms for general linear groups over the function field $\mathbb{F}_{q}(X)$, and the Hall multiplication encodes the action of Hecke operators. Here, the theory makes contact with the Langlands program over function fields (for more on this, see the beautiful papers [14,15]).

1.2 Hall algebras in a non-additive setting. A closer examination of the basic construction of $\mathrm{H}_{\mathcal{C}}$ outlined above shows that the assumption that $\mathcal{C}$ be additive is unnecessary. All that is needed to make sense of the Hecke correspondence (1) used to define $\mathrm{H}_{\mathcal{C}}$ is a category with a well-behaved notion of exact sequences. In the important paper [6], the notion of proto-exact category is introduced as a non-additive generalization of the notion of Quillen exact category above, and shown to suffice for the construction of an associative Hall algebra. As in the additive case, such a category is defined in terms of a pair $(\mathfrak{M}, \mathfrak{E})$ of admissible mono/epis which are required to satisfy certain properties. The simplest example of a non-additive proto-exact category is the category $\mathcal{S}$ et $\bullet$ of finite pointed sets, with $\mathfrak{M}$ all pointed injections, and $\mathfrak{E}$ those pointed surjections which are isomorphisms away from the base-point.

Many examples of non-additive proto-exact categories $\mathcal{C}$ arise in combinatorics. Here, $\mathrm{Ob}(\mathcal{C})$ typically consist of combinatorial structures equipped with operations of "inserting" and "collapsing" of sub-structures, corresponding to $(\mathfrak{M}, \mathfrak{E})$. Examples of such $\mathcal{C}$ include trees, graphs, posets, semigroup representations in $\mathcal{S e t}_{\bullet}$, quiver representations in $\mathcal{S}_{\text {et }}$ etc. (see $[17,23-26]$ ). The product in $\mathrm{H}_{\mathcal{C}}$, which counts all extensions between two objects, thus amounts to enumerating all combinatorial structures that can be assembled from two others. Here $\mathrm{H}_{\mathcal{C}}$ is typically (dual to) a combinatorial Hopf algebra in the sense of [18]. Many combinatorial Hopf algebras arise via this mechanism, including the Hopf algebra of symmetric functions, the Connes-Kreimer Hopf algebras of rooted trees and Feynman graphs, and many others. 
1.3 The Waldhausen $\mathcal{S}$-construction and K-theory of proto-exact categories. It is a natural question what advantages, if any, there are to thinking of combinatorial objects in terms of proto-exact categories and Hall algebras. The answer, as evident in other forms of categorification, is that certain constructions are only visible at the categorical level. In [6], the authors associate to a proto-exact category $\mathcal{C}$ a simplicial groupoid $\mathcal{S} . \mathcal{C}$, called the Waldhausen $\mathcal{S}$-construction, where $\mathcal{S}_{n} \mathcal{C}$ is closely related to $\mathbf{F L}_{n}$ above. $\mathcal{S}_{\bullet} \mathcal{C}$ is shown to have a number of very interesting properties, including the structure of a 2Segal space - a form of higher associativity, of which $\mathrm{H}_{\mathcal{C}}$ is but a shadow. This structure was also studied in the papers [8-10] from a somewhat different perspective.

As explained in [6], $\mathcal{S} \cdot \mathcal{C}$ may be used to define the algebraic K-theory of $\mathcal{C}$, by

$$
K_{n}(\mathcal{C})=\pi_{n+1}\left|\mathcal{S}_{\bullet} \mathcal{C}\right|
$$

where $\left|\mathcal{S}_{\bullet} \mathcal{C}\right|$ denotes the geometric realization of $S_{\bullet} \mathcal{C}$. These groups appear to contain interesting homotopy-theoretic information, even for very simple categories like $\mathcal{S e t}_{\bullet}$, as evidenced by the following result:

Theorem $1.1([2,4]) . K_{*}\left(\mathcal{S e t}_{\bullet}\right) \simeq \pi_{*}^{S}(\mathrm{~S})$, where the right hand side denotes the stable homotopy groups of the sphere spectrum.

1.4 Matroids as a proto-exact category. Matroids are combinatorial structures which abstract different notions of independence encountered across mathematics. A matroid $M$ consists of a finite set $E_{M}$ (the ground set) together with a collection of independent subsets $I \subseteq 2^{E_{M}}$ satisfying certain natural properties. The prototypical example is obtained by taking $E_{M}$ to be a set of vectors in some vector space $V$, and taking $I \subseteq 2^{E_{M}}$ to be those subsets which are linearly independent. Matroids and their generalizations have found a vast array of applications across several areas of mathematics, such as for instance in tropical geometry, where valuated matroids play the role of linear spaces.

Matroids form a category Mat. with respect to strong maps, which are a generalization of linear map in this setting, and it is the category Mat. that forms the object of study in this paper. Other aspects of Mat. have also been studied in [12]. For technical reasons, we prefer to work with pointed matroids, where the ground set $E_{M}$ is pointed by a distinguished element. We show

Theorem A ( $\$ 4$ and $\S 5)$. The category Mat. of pointed matroids and strong maps has the structure of a finitary proto-exact category, with $\mathfrak{M}$ matroid restrictions and $\mathfrak{E}$ matroid contractions.

We give two proofs of this theorem, which reduces to verifying the existence of certain special pushouts/pull-backs in Mat.. The first is written in the "classical" language of matroids, while the second uses a description of Mat. in terms of embedded semimodules over the Boolean semiring $\mathbb{B}$ given in [3]. We are hopeful that a description of 
valuated matroids along the lines of [3] can be given, and that our proof should generalize to that situation as well.

We proceed to define and study the algebraic K-theory of Mat• via Definition 6.1. Mat. has an exact forgetful functor to $\mathcal{S e t}_{\bullet}$ possessing an exact left adjoint sending $E \in \mathcal{S}_{\text {et }}$ to the "free pointed matroid on $E^{\prime}$. These can be used to relate the $K_{*}\left(\mathcal{S}_{e t} t_{\bullet}\right)$ and $K_{*}\left(\mathbf{M a t}_{\bullet}\right)$. We show:

Theorem B (Theorem 6.3). $K_{0}$ (Mat.) $\simeq \mathbb{Z} \oplus \mathbb{Z}$

and

Theorem C (Theorem 6.4). There are injective group homomorphisms

$$
\pi_{n}^{\text {s }}(\mathrm{S}) \simeq K_{n}\left(\mathcal{S e t}_{\bullet}\right) \hookrightarrow K_{n}\left(\text { Mat }_{\bullet}\right)
$$

for all $n \geq 0$.

This shows in particular that $K_{n}$ (Mat.) is in general non-trivial for $n \geq 0$.

As a corollary of Theorem A, we are able to define the Hall algebra $\mathrm{H}_{\text {Mat. }}$. The product $[M] \star[N]$ in this algebra enumerates all matroids $[L]$ such that $L \mid S \simeq M$ and $L / S \simeq N$ for some subset $S \subseteq E_{L}$. $\mathrm{H}_{\text {Mat. }}$ turns out to be dual to combinatorial Hopf algebra introduced by Schmitt in [22], called the matroid-minor Hopf algebra. We obtain the following:

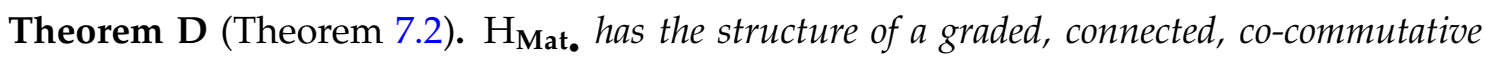
Hopf algebra, dual to Schmitt's matroid-minor Hopf algebra.

1.5 Outline of this paper. In section 2 we recall the basics of proto-exact categories and their Hall algebras following [6]. Basic notions regarding pointed matroids are laid out in section 3. Sections 4 and 5 contain two proofs of Theorem A - one in classical matroidtheoretic language and one using the language of $\mathbb{B}$-modules introduced in [3]. In section 6 we define the K-theory of Mat. and prove Theorems B and C. Finally, in section 7.1 we relate the Hall algebra $\mathrm{H}_{\text {Mat. }}$ to Schmitt's matroid-minor Hopf algebra, proving Theorem D.

\section{Acknowledgments}

The third author is grateful to Tobias Dyckerhoff for explanations regarding the paper [6] and for the support of a Simons Foundation Collaboration Grant. 


\section{PROTO-EXACt CATEGORIES AND THEIR HALl ALGEBRAS}

In this section, we recall the notion of a proto-exact category $\mathcal{E}$ following [6], where we direct the interested reader for details and proofs. This is a generalization of a Quillen exact category that allows $\mathcal{E}$ to be non-additive, and yet provides enough structure to define an associative Hall algebra by counting certain distinguished exact sequences in $\mathcal{E}$. As usual, we denote monomorphisms in $\mathcal{E}$ by $\hookrightarrow$ and epimorphisms by $\rightarrow$.

A commutative square

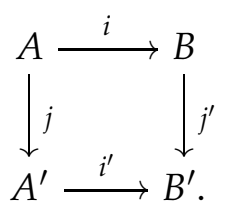

is called biCartesian if it is both Cartesian and co-Cartesian.

Definition 2.1. A proto-exact category is a category $\mathcal{E}$ equipped with two classes of morphisms $\mathfrak{M}, \mathfrak{E}$, called admissible monomorphisms and admissible epimorphisms respectively. The triple $(\mathcal{E}, \mathfrak{M}, \mathfrak{E})$ is required to satisfy the following properties:

(1) The category $\mathcal{E}$ has a zero object 0 . Any morphism $0 \rightarrow A$ is in $\mathfrak{M}$, and any morphism $A \rightarrow 0$ is in $\mathfrak{E}$.

(2) The classes $\mathfrak{M}, \mathfrak{E}$ are closed under composition and contain all isomorphisms.

(3) A commutative square (6) in $\mathcal{E}$ with $i, i^{\prime} \in \mathfrak{M}$ and $j, j^{\prime} \in \mathfrak{E}$ is Cartesian iff it is co-Cartesian.

(4) Every diagram in $\mathcal{E}$

$$
A^{\prime} \stackrel{i^{\prime}}{\longrightarrow} B^{\prime} \stackrel{j^{\prime}}{\longleftarrow} B
$$

with $i^{\prime} \in \mathfrak{M}$ and $j^{\prime} \in \mathfrak{E}$ can be completed to a biCartesian square (6) with $i \in \mathfrak{M}$ and $j \in \mathfrak{E}$.

(5) Every diagram in $\mathcal{E}$

$$
A^{\prime} \stackrel{j}{\longleftarrow} A \stackrel{i}{\longleftrightarrow} B
$$

with $i \in \mathfrak{M}$ and $j \in \mathfrak{E}$ can be completed to a biCartesian square (6) with $i^{\prime} \in \mathfrak{M}$ and $j^{\prime} \in \mathfrak{E}$.

A biCartesian square of the form with the horizontal maps are in $\mathfrak{M}$ and the vertical maps are in $\mathfrak{E}$

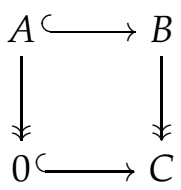


is called an admissible short exact sequence, or, an admissible extension of $C$ by $A$, and will also be written as

$$
A \hookrightarrow B \rightarrow C
$$

We will denote the object $C$ (unique up to a unique isomorphism) by $B / A$. A functor $F: \mathcal{C} \mapsto \mathcal{D}$ between proto-exact categories is exact if it preserves admissible short exact sequences. Two extensions $A \hookrightarrow B \rightarrow C$ and $A \hookrightarrow B^{\prime} \rightarrow C$ of $C$ by $A$ are called equivalent if there exists a commutative diagram

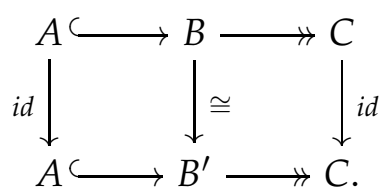

and the set of equivalence classes of such will be denoted by $\operatorname{Ext}_{\mathcal{E}}(C, A)$. Two admissible monomorphisms $i_{1}: A \hookrightarrow B$ and $i_{2}: A^{\prime} \hookrightarrow B$ in $\mathfrak{M}$ are isomorphic if there exists an isomorphism $f: A \rightarrow A^{\prime}$ such that $i_{1}=i_{2} \circ f$. We call the isomorphism classes in $\mathfrak{M}$ admissible subobjects.

Definition 2.2. A proto-exact category $\mathcal{E}$ is called finitary if, for every pair of objects $A, B$, the sets $\operatorname{Hom}_{\mathcal{E}}(A, B)$ and $\operatorname{Ext}_{\mathcal{E}}(A, B)$ have finite cardinality.

Example 2.3. (1) Any Quillen exact category is proto-exact, with the same exact structure. In particular, any Abelian category $\mathcal{E}$ is proto-exact with $\mathfrak{M}$ all monomorphisms and $\mathfrak{E}$ all epimorphisms respectively. The category $\operatorname{Rep}\left(Q, \mathbb{F}_{q}\right)$ of representations of a quiver $Q$ over a finite field $\mathbb{F}_{q}$, and $\operatorname{Coh}\left(X / \mathbb{F}_{q}\right)$ - the category of coherent sheaves on a smooth projective variety over $\mathbb{F}_{q}$ are both finitary Abelian.

(2) The simplest example of a non-additive proto-exact category is the category $\mathcal{S} e t_{\bullet}$ whose objects are pointed sets with pointed maps as morphisms. Here $\mathfrak{M}$ consists of all pointed injections, and $\mathfrak{E}$ all pointed surjections $p:(S, *) \rightarrow(T, *)$ such that $\left.p\right|_{S \backslash p^{-1}(*)}$ is injective. The full subcategory $\mathcal{S e t}_{\bullet}^{f i n}$ of finite pointed sets is finitary.

2.1 The Hall algebra. Let $\mathcal{E}$ be a finitary proto-exact category, and $k$ a field of characteristic zero. Define the Hall algebra $\mathrm{H}_{\mathcal{E}}$ over $k$ as

$$
\mathrm{H}_{\mathcal{E}}:=\{f: \operatorname{Iso}(\mathcal{E}) \rightarrow k \mid f \text { has finite support }\},
$$

where $\operatorname{Iso}(\mathcal{E})$ denotes the set of isomorphism classes in $\mathcal{E}$. $\mathrm{H}_{\mathcal{E}}$ is an associative $k$-algebra under the convolution product

$$
f \bullet g([B]):=\sum_{A \subseteq B} f([B / A]) g([A]),
$$

where the summation $\sum_{A \subseteq B}$ is taken over isomorphism classes of admissible sub-objects $i: A \hookrightarrow B, i \in \mathfrak{M}$, and $[A]$ etc. denotes the isomorphism class of $A$ in $\mathcal{E}$. Note that this 
sum is finite, since $\mathcal{E}$ is assumed finitary. $\mathrm{H}_{\mathcal{E}}$ has a basis consisting of delta-functions $\delta_{[B]}$, $[B] \in \operatorname{Iso}(\mathcal{E})$, where

$$
\delta_{[B]}([A])= \begin{cases}1 & A \simeq B \\ 0 & \text { otherwise } .\end{cases}
$$

The multiplicative unit of $\mathrm{H}_{\mathcal{E}}$ is given by $\delta_{[0]}$. The structure constants of this basis are given by

$$
\delta_{[A]} \bullet \delta_{[C]}=\sum_{[B] \in \operatorname{Iso}(\mathcal{E})} g_{A, C}^{B} \delta_{[B]}
$$

where

$$
g_{A, C}^{B}=\#\{D \subseteq B \mid D \simeq C, B / D \simeq A\} .
$$

In other words, $g_{A, C}^{B}$ counts the number of admissible subobjects $D$ of $B$ isomorphic to $C$ such that $B / D$ is isomorphic to $A$. The Grothendieck group of $\mathcal{E}$, denoted $K_{0}(\mathcal{E})$ is defined as the free group on $\operatorname{Iso}(\mathcal{E})$ modulo the relations $[B]=[A][C]$ for every admissible short exact sequence (7). When $\mathcal{E}$ admits split admissible short exact sequences of the form

$$
A \hookrightarrow A \oplus B \rightarrow B
$$

$K_{0}(\mathcal{E})$ is Abelian, and has the familiar description

$$
K_{0}(\mathcal{E})=\mathbb{Z}[\operatorname{Iso}(\mathcal{E})] / \sim,
$$

where $\sim$ is generated by the relations $[B]=[A]+[C]$ for all admissible short exact sequences (7). We denote by $K_{0}(\mathcal{E})^{+} \subseteq K_{0}(\mathcal{E})$ the sub-semigroup generated by the effective classes. $\mathrm{H}_{\mathcal{E}}$ is naturally graded by $K_{0}(\mathcal{E})^{+}$, with $\operatorname{deg}\left(\delta_{[B]}\right)=[B] \in K_{0}(\mathcal{E})^{+}$.

Whether $\mathrm{H}_{\mathcal{E}}$ carries a co-product making it into a bialgebra depends on further properties of $\mathcal{E}$. For instance, if $\mathcal{E}$ is finitary, Abelian, linear over $\mathbb{F}_{q}$ and hereditary, $\mathrm{H}_{\mathcal{E}}$ carries the so-called Green's co-product (see [21]). In this paper, we will be concerned with situations where $\mathcal{E}$ is not additive, and where the following alternative construction applies. Suppose that $\mathcal{E}$ is equipped with finite direct sums, and that the only admissible subobjects of $A \oplus B$ are of the form $A^{\prime} \oplus B^{\prime}$, where $A^{\prime} \subseteq A, B^{\prime} \subseteq B$. In this case, we may define

$$
\Delta: \mathrm{H}_{\mathcal{E}} \rightarrow \mathrm{H}_{\mathcal{E}} \otimes \mathrm{H}_{\mathcal{E}}
$$

by

$$
\Delta(f)([A],[B])=f([A \oplus B]) .
$$

$\Delta$ is easily seen to be compatible with the associative product $\bullet$ on $\mathrm{H}_{\mathcal{E}}$. It is clear from (9) that $\Delta$ is co-commutative, and that the subspace of primitive elements of $\mathrm{H}_{\mathcal{E}}$ is spanned by $\left\{\delta_{[B]}\right\}$, where $B$ is indecomposable (i.e. cannot be written as a non-trivial direct sum of 
sub-objects). By the Milnor-Moore theorem, any graded connected co-commutative bialgebra is a Hopf algebra, isomorphic to the enveloping algebra of its primitive elements. To summarize, we have:

Theorem 2.4. Let $\mathcal{E}$ be a finitary proto-exact category $\mathcal{E}$ satisfying the condition

$$
C \subseteq A \oplus B \Rightarrow C \simeq A^{\prime} \oplus B^{\prime}, A^{\prime} \subseteq A, B^{\prime} \subseteq B
$$

Then $\mathrm{H}_{\mathcal{E}}$ has the structure of a $K_{0}^{+}(\mathcal{E})$-graded, connected, co-commutative Hopf algebra over $k$ with co-product (9). $\mathrm{H}_{\mathcal{E}} \simeq \mathbf{U}\left(\delta_{[B]}\right)$, where $B$ is indecomposable.

Example 2.5. The category $\mathcal{E}=\mathcal{S e t}_{\bullet}$, also known as the category of finite dimensional vector spaces over "the field with one element $\mathbb{F}_{1}$ " has direct sums (defined as wedge sums), and satisfies the conditions of the theorem. In this case $\mathrm{H}_{\mathcal{E}} \simeq k[x]$, with

$$
\Delta(x)=x \otimes 1+1 \otimes x
$$

\section{Matroids And Strong Maps}

This section provides a short introduction to the basic terminology and results of matroid theory we use in this paper. For more details, the reader is encouraged to see [20].

Matroids are combinatorial abstractions of various properties of linear independence among finitely many vectors in a vector space. As such, these objects admit a number of "cryptomorphic" axiomatizations; that is, there are a variety of ways of formulating the axioms for matroids, any of which can be translated to any other. We present one such formulation, namely the flats operator. Given a finite set $E$ (the ground set of our matroid), a function $\sigma: 2^{E} \rightarrow 2^{E}$ is a flats operator for a matroid on $E$ when it satisfies the following axioms:

(F1) For all $S \subseteq E$ we have $S \subseteq \sigma S$.

(F2) The map $\sigma$ is idempotent (i.e. $\sigma=\sigma \sigma$ ).

(F3) For all $S \subseteq T \subseteq E$ we have $\sigma S \subseteq \sigma T$.

(F4) For all $S \subseteq E$ and all $x \in E$ and $y \in \sigma(S \cup\{x\}) \backslash \sigma S$ we have $x \in \sigma(S \cup\{y\})$.

Sets of the form $\sigma S$ for some $S \subseteq E$ are called flats.

Remark 3.1. Note that (F1), (F2), and (F3) are the axioms of a closure operator on $E$. Thus a flats operator on $E$ is a closure operator on $E$ satisfying $(\mathrm{F} 4)$.

Example 3.2. The following are the prototypical examples of flats operators. ${ }^{1}$

(1) Let $E$ be a finite subset of a vector space $V$. For all $S \subseteq E$ let $\sigma S=\langle S\rangle \cap E$ where $\langle S\rangle$ denotes the the subspace of $V$ spanned by $S$. Then $\sigma$ is a flats operator on $E$.

\footnotetext{
${ }^{1}$ Not all matroids arise in this way. For more details, see [20].
} 
(2) Let $\Gamma$ be a graph with a finite edge set $E$. For all $S \subseteq E$ let $\sigma S$ be the set all edges $e$ for which there is a path in $\Gamma$ connecting the ends of $e$ and using only edges of $S$. Then $\sigma$ is a flats operator on $E$.

Remark 3.3. We note the following:

(1) In light of Example 3.2, this structure on $E$ abstracts the notion of "span" in a vector space.

(2) Given a flats operator $\sigma$ for a matroid $M$ on $E$, one can construct $\mathcal{F}(M)$, the set of all flats determined by $\sigma$. The set of flats can also be used to axiomatize matroids "cryptomorphically," but the details are unimportant for the results of this paper. We use this fact implicitly below when we define the restriction and contraction.

In what follows, for a matroid $M$ on a finite set $E$, we let $\mathcal{F}(M)$ be the set of all flats of M.

Definition 3.4. Let $M$ be a matroid on $E$ and $S \subseteq E$.

(1) The restriction of $M$ to $S$ is the matroid $M \mid S$ on $S$ with flats

$$
\mathcal{F}(M \mid S)=\{A \cap S \mid A \in \mathcal{F}(M)\} .
$$

(2) The contraction of $M$ by $S$ is the matroid $M / S$ on $E \backslash S$ with flats

$$
\mathcal{F}(M / S)=\{A \backslash S \mid S \subseteq A \in \mathcal{F}(M)\} .
$$

Example 3.5. Let $M$ be a matroid on a ground set $E$ and $S \subseteq E$.

(1) If $M$ is obtained from $E \subseteq V$ for a vector space $V$ as in Example 3.2, then $M \mid S$ is obtained likewise from $S$ in $\langle S\rangle$. In particular, matroid restriction corresponds to restriction to a subspace in the context of vector spaces.

(2) If $M$ is obtained from $E$ as the edge set of a graph $\Gamma$ as in Example 3.2, then $M \mid S$ is obtained likewise from the graph $\Gamma \mid S$ with only the edges of $S$.

Example 3.6. Let $M$ be a matroid on a ground set $E$ and $S \subseteq E$.

(1) If $M$ is obtained from $E \subseteq V$ for a vector space $V$ as in Example 3.2, then $M / S$ is obtained likewise from $E \backslash S$ in the vector space $V /\langle S\rangle$. In particular, matroid contraction corresponds to the quotient by a subspace in the context of vector spaces.

(2) If $M$ is obtained from $E$ as the edge set of a graph $\Gamma$ as in Example 3.2, then $M / S$ is obtained likewise from the contracted graph $\Gamma / S$ in which the edges of $S$ are contracted.

Definition 3.7. The direct sum $M_{1} \oplus M_{2}$ of matroids $M_{1}$ and $M_{2}$ is defined as the matroid on the ground set $E_{M_{1}} \sqcup E_{M_{2}}$ with flats operator $\sigma_{1} \sqcup \sigma_{2}$. In other words, the flats of $M_{1} \oplus M_{2}$ are of the form $F_{1} \sqcup F_{2}$ where $F_{1} \in \mathcal{F}\left(M_{1}\right)$ and $F_{2} \in \mathcal{F}\left(M_{2}\right)$. 
Example 3.8. (1) If $M_{1}, M_{2}$ are obtained from subsets $E_{i} \subseteq V_{i}, i=1,2$ for vector spaces $V_{1}, V_{2}$, then $M_{1} \oplus M_{2}$ is obtained from $E_{1} \sqcup E_{2} \subseteq V_{1} \oplus V_{2}$.

(2) If $M_{1}, M_{2}$ are graphic, corresponding to graphs $\Gamma_{1}, \Gamma_{2}$, then $M_{1} \oplus M_{2}$ is the graphic matroid of the disjoint union $\Gamma_{1} \sqcup \Gamma_{2}$.

A loop of a matroid is an element of the ground set which belongs to the flat $\sigma \varnothing .^{2} \mathrm{~A}$ pointed matroid is a pair $\left(M, *_{M}\right)$ where $M$ is a matroid and $*_{M}$ is a distinguished loop (a.k.a. the point). We think of the point as a zero element. We will often suppress the point in our notation and say that $M$ is a pointed matroid with a point $*_{M}$.

Remark 3.9. When dealing with pointed matroids $\left(M, *_{M}\right)$, we adopt the following conventions to simplify notation, and to ensure that the result of various operations is also a pointed matroid. Recall that the coproduct of pointed sets $\left(S_{1}, p_{1}\right),\left(S_{2}, p_{2}\right)$ is $S_{1} \vee S_{2}$ their wedge sum, in which the basepoints get identified.

(1) We will denote by $\widetilde{E}_{M}$ the non-zero elements in the ground set. Thus for a pointed matroid $\left(M, *_{M}\right)$ we have $E_{M}=\widetilde{E}_{M} \sqcup\left\{*_{M}\right\}$.

(2) For $S \subseteq \widetilde{E}_{M}, M \mid S$ will denote the restriction $M \mid\left(S \cup\left\{*_{M}\right\}\right)$. Since $*_{M} \notin S$, the definition of $M / S$ needs no modification.

(3) If $\left(M_{1}, *_{M_{1}}\right),\left(M_{2}, *_{M_{2}}\right)$ are pointed matroids, then $M_{1} \oplus M_{2}$ is defined as the pointed matroid on the ground set $E_{M_{1}} \vee E_{M_{2}}$ with the flats operator $\sigma_{1} \vee \sigma_{2}$.

Having the notion that pointed matroids are generalizations of vector spaces, one naturally seeks an appropriate translation of linear maps to this setting.

Definition 3.10. Let $M$ and $N$ be pointed matroids on ground sets $E_{M}$ and $E_{N}$ respectively and having flats $\mathcal{F}_{M}$ and $\mathcal{F}_{N}$ respectively. A (pointed) strong map of pointed matroids $f: M \rightarrow N$ is a function $f: E_{M} \rightarrow E_{N}$ such that $f\left(*_{M}\right)=*_{N}$ and for all $A \in \mathcal{F}_{N}$ we have $f^{-1} A \in \mathcal{F}_{M}$.

Example 3.11. The following are the prototypical examples of strong maps. ${ }^{3}$

(1) Let $M$ and $N$ be pointed matroids arising from $k$-vector spaces $V_{M}$ and $V_{N}$ respectively for some common field $k$ as in Example 3.2 above. Every linear map $L: V_{M} \rightarrow V_{N}$ determines a (pointed) strong map $M \rightarrow N$.

(2) Let $\Gamma$ and $\Lambda$ be graphs with distinguished loops $*_{\Gamma}$ and $*_{\Lambda}$, and let $\left(M_{\Gamma}, *_{\Gamma}\right)$ and $\left(M_{\Lambda}, *_{\Lambda}\right)$ be the pointed matroids arising from $\Gamma$ and $\Lambda$ as in Example 3.2 above. Every graph morphism $f: \Gamma \rightarrow \Lambda$ preserving the points yields a pointed strong $\operatorname{map} M_{\Gamma} \rightarrow M_{\Lambda}$.

\footnotetext{
${ }^{2}$ In a matroid determined by a graph as in Example 3.2.2, this is exactly a loop of the graph.

${ }^{3}$ Not all strong maps between matroids arise in this way (even if the matroids themselves arise in this fashion).
} 
The following is immediate:

Proposition 3.12. Pointed matroids and strong maps together form a category Mat..

There is a forgetful functor

$$
\mathbb{F}: \text { Mat• } \mapsto \text { Set• }
$$

which takes a pointed matroid $\left(M, *_{M}\right)$ to its underlying pointed ground set $\left(E_{M}, *_{M}\right)$.

\section{Mat. AS A PROTO-EXACT CATEGORY}

Our goal in this section is to show that Mat. has the structure of a proto-exact category in the sense of [6]. We begin by exhibiting the classes of admissible monos/epis.

Definition 4.1. Let $\mathfrak{M}$ consist of all strong maps in Mat. that can be factored as

$$
N \stackrel{\sim}{\rightarrow} M \mid S \hookrightarrow M,
$$

and $\mathfrak{E}$ consists of all strong maps in Mat. that can be factored as

$$
M \rightarrow M / S \stackrel{\sim}{\rightarrow} N
$$

for some $S \subseteq E_{M}$.

Throughout this section, we let $\mathcal{E}:=$ Mat. . We show that $(\mathcal{E}, \mathfrak{M}, \mathfrak{E})$ as above is a protoexact category. We first prove certain basic lemmas which will be used in what follows. For the notational convenience, for a function $f: A \rightarrow B$ and $S \subseteq A$, we will write $f S$ for the image $f(S)$ whenever there is no possible confusion. All matroids are assumed to be pointed unless otherwise stated.

Lemma 4.2. Let $M($ resp. $N)$ be a pointed matroid on $E_{M}$ (resp. $\left.E_{N}\right)$. A function $f: E_{M} \rightarrow E_{N}$ is an isomorphism in $\mathcal{E}$ precisely when $f$ is a pointed bijection satisfying

$$
A \in \mathcal{F}(M) \Longleftrightarrow f A \in \mathcal{F}(N) \text {. }
$$

Proof. Let $f: E_{M} \rightarrow E_{N}$ be a set map.

Sufficiency: Suppose $f$ is an isomorphism of pointed matroids $M$ and N. Applying the forgetful functor $\mathbb{F}$ we see that $f$ is necessarily a pointed bijection; moreover, the underlying map of the inverse map $f^{-1}$ is the inverse of the underlying map. Thus both $f$ and $f^{-1}$ are strong maps; given $f A \in \mathcal{F}(N)$ we have $f^{-1}(f A)=A \in \mathcal{F}(M)$, and similarly given $A \in \mathcal{F}(M)$ we have $\left(f^{-1}\right)^{-1} A=f A \in \mathcal{F}(N)$.

Necessity: Suppose $f$ is a pointed bijection with $A \in \mathcal{F}(M)$ if and only if $f A \in \mathcal{F}(N)$. Notice that we need only see $f$ and $f^{-1}$ are strong maps to conclude. Given $F \in \mathcal{F}(N)$ we have $F=f\left(f^{-1} F\right)$, so $f^{-1} F \in \mathcal{F}(M)$ by our assumption; thus $f$ is a strong map. Moreover, given $F \in \mathcal{F}(M)$ we have $\left(f^{-1}\right)^{-1} F=f F \in \mathcal{F}(N)$; thus $f^{-1}$ is a strong map, and $f$ is an isomorphism. 
Notice that given subsets $T \subseteq S \subseteq E_{M}$ we have the following:

$$
\mathcal{F}((M \mid S) / T)=\{(F \cap S) \backslash T \mid T \subseteq F \in \mathcal{F}(M)\} .
$$

Lemma 4.3. Let $f: M \rightarrow N$ be an isomorphism in $\mathcal{E}$.

(1) For all $*_{M} \in S \subseteq E_{M}$ the map $\left.f\right|_{S}: M|S \rightarrow N| f S$ is an isomorphism.

(2) For all $S \subseteq E_{M} \backslash\left\{*_{M}\right\}$ the map $\left.f\right|_{E_{M} \backslash S}: M / S \rightarrow N / f S$ is an isomorphism.

Proof. Let $f: M \rightarrow N$ be an isomorphism in $\mathcal{E}$. Note that $f\left(*_{M}\right)=*_{N}$, so we need not worry about contracting or deleting the point as $f$ is a bijection.

Restriction: We have the following string of equivalent statements via Definition 3.4:

$$
\begin{aligned}
A \in \mathcal{F}(M \mid S) & \Longleftrightarrow \exists B \in \mathcal{F}(M) \text { such that } A=B \cap S \\
& \Longleftrightarrow \exists B \in \mathcal{F}(M) \text { such that } f A=f B \cap f S \\
& \Longleftrightarrow \exists B^{\prime} \in \mathcal{F}(N) \text { such that } f A=B^{\prime} \cap f S \\
& \Longleftrightarrow f A \in \mathcal{F}(N \mid f S)
\end{aligned}
$$

Moreover $\left.f\right|_{S}$ is a pointed bijection. Hence $\left.f\right|_{S}$ is an isomorphism by Lemma 4.2.

Contraction: Similarly, we have the following equivalent statements via Definition 3.4:

$$
\begin{aligned}
A \in \mathcal{F}(M / S) & \Longleftrightarrow \exists B \in \mathcal{F}(M) \text { such that } S \subseteq B \text { and } A=B \backslash S \\
& \Longleftrightarrow \exists B \in \mathcal{F}(M) \text { such that } f S \subseteq f B \text { and } f A=f B \backslash f S \\
& \Longleftrightarrow \exists B^{\prime} \in \mathcal{F}(N) \text { such that } f S \subseteq B^{\prime} \text { and } f A=B^{\prime} \backslash f S \\
& \Longleftrightarrow f A \in \mathcal{F}(N / f S)
\end{aligned}
$$

Moreover $\left.f\right|_{E_{M} \backslash S}$ is a pointed bijection. Hence $\left.f\right|_{E_{M} \backslash S}$ is an isomorphism by Lemma 4.2.

Lemma 4.4. For every matroid $M$ and every $T \subseteq S \subseteq E_{M}$ we have $(M \mid S) / T=(M / T) \mid S$.

Proof. It is a standard exercise that $(M \backslash A) / B=(M / B) \backslash A$. Apply this result with $A=E \backslash S$ and $B=T$.

Lemma 4.5. Let $f: M \rightarrow N$ be a matroid strong map. Then we have the following:

(1) $f$ is a monomorphism in $\mathcal{E}$ precisely when $f$ is injective.

(2) $f$ is an epimorphism in $\mathcal{E}$ precisely when $f$ is surjective.

Proof. The necessity must hold for both statements simply by noting that every strong map is also a function on underlying sets, and thus the required properties for monics and epics must hold by the corresponding properties of their underlying maps.

Assume $f: M \rightarrow N$ is monic and consider the pointed matroid $U_{1,1}^{*}=(\{1, *\}, *)$ with flats $\{*\}$ and $\{1, *\}$. Trivially every pointed map $g: U_{1,1}^{*} \rightarrow M$ is a strong map. For each 
$a \in E_{M}$ let $g_{a}: U_{1,1}^{*} \rightarrow M$ denote the pointed map sending $1 \mapsto a$. Suppose $f(a)=f(b)$ for some $a, b \in E_{M}$; thus $f g_{a}=f g_{b}$ yields $a=g_{a}(1)=g_{b}(1)=b$ by the assumption that $f$ is monic. Hence $f$ is injective as desired.

Assume $f: M \rightarrow N$ is epic and consider the pointed matroid $U_{0,1}^{*}=(\{1, *\}, *)$ with the flat $\{1, *\}$. Trivially every pointed map $h: N \rightarrow U_{0,1}^{*}$ is a strong map. For each $a \in N$ define $h_{a}: N \rightarrow U_{0,1}^{*}$ by $x \mapsto 1$ precisely when $x=a$. If $f$ is not surjective, then choosing any $a \in E_{N} \backslash f E_{M}$ we have $h_{a} f=h_{*} f$, and thus $h_{*}=h_{a}$ by the assumption that $f$ is epic. But this implies $a=* \in f E_{M}$, which is absurd. Hence $f$ is surjective as desired.

Lemma 4.6. Let $M$ be a matroid on $E_{M}$ and $S \subseteq E_{M}$.

(1) If $*_{M} \in S$, then there is a canonical map $i_{S}: M \mid S \hookrightarrow M$ in $\mathcal{E}$.

(2) If $*_{M} \notin S$, then there is a canonical map $c_{S}: M \rightarrow M / S$ in $\mathcal{E}$.

Proof. Restriction: Suppose $*_{M} \in S$ and let $i_{S}$ denote the inclusion $S \hookrightarrow E_{M}$. Note that for all $F \in \mathcal{F}(M)$ we have $i_{S}^{-1} F=S \cap F \in \mathcal{F}(M \mid S)$ by Definition 3.4. Hence $i_{S}$ is a strong map as desired.

Contraction: Suppose $*_{M} \notin S$ and let $c_{S}: E_{M} \rightarrow E_{M} \backslash S$ denote the map defined by $c_{S} S=\{*\}$ and $\left.c_{S}\right|_{E_{M} \backslash S}=$ id. Now for all $F \in \mathcal{F}(M / S)$, there is a flat $A \in \mathcal{F}(M)$ with $S \subseteq A$ and $F=A \backslash S$. Thus noting $* \in F$ yields $c_{S}^{-1} F=S \cup F=S \cup(A \backslash S)=A \in \mathcal{F}(M)$ as $S \subseteq A$. Hence $c_{S}$ is a strong map as desired.

Now, we prove that $(\mathcal{E}, \mathfrak{M}, \mathfrak{E})$ is a proto-exact category by verifying each property as below.

Proposition 4.7 (Verifying Property 1$)$. $(\mathcal{E}, \mathfrak{M}, \mathfrak{E})$ is equipped with a zero object.

Proof. The pointed matroid $(\{*\}, *)$ is the zero object. Indeed, one easily sees that every map $0 \rightarrow M$ can be factored as $0 \rightarrow M \mid\{*\} \hookrightarrow M$ and hence is in $\mathfrak{M}$. Similarly, every map $M \rightarrow 0$ can be factored as $M \rightarrow M /\left(E_{M} \backslash\left\{*_{M}\right\}\right) \rightarrow 0$ and hence in $\mathfrak{E}$.

Proposition 4.8 (Verifying Property 2). The classes $\mathfrak{M}$ and $\mathfrak{E}$ are closed under composition and contain all isomorphisms.

Proof. To see Property 2, first notice that every isomorphism $f: M \rightarrow N$ can be factored as $M \stackrel{f}{\rightarrow} N \mid E_{N} \hookrightarrow N$ and $M \rightarrow M / \varnothing \stackrel{f}{\rightarrow} N$; in particular, every isomorphism is a member of both $\mathfrak{M}$ and $\mathfrak{E}$.

Given two composible members $f: M \rightarrow N$ and $g: N \rightarrow P$ of $\mathfrak{M}$, factor these as $M \stackrel{f_{0}}{\rightarrow} N \mid S \hookrightarrow N$ and $N \stackrel{g_{0}}{\rightarrow} P \mid T \hookrightarrow P$. Now the composite $g f$ factors as $M \stackrel{f_{0}}{\rightarrow} N \mid S \hookrightarrow$ $N \stackrel{g_{0}}{\rightarrow} P \mid T \hookrightarrow P$. Notice by construction that $g_{0} S \subseteq T$; as $g_{0}$ is an isomorphism, so Lemma 4.3 yields that $\left.g_{0}\right|_{S}: N|S \rightarrow P| g_{0} S$ is an isomorphism. In particular, we have the factorization $M \stackrel{f_{0}}{\rightarrow} N|S \stackrel{\text { id }}{\rightarrow} N| S \stackrel{g_{0} \mid s}{\longrightarrow} P \mid g_{0} S \hookrightarrow P$; the first three arrows in this diagram 
are isomorphisms. Hence $M \stackrel{\left.g_{0}\right|_{s} f}{\longrightarrow} P \mid g_{0} S \hookrightarrow P$ is a factorization of $g f$ which shows this is an admissible monomorphism.

Given two composible members $f: M \rightarrow N$ and $g: N \rightarrow P$ of $\mathfrak{E}$, factor these as $M \rightarrow M / S \stackrel{f_{0}}{\rightarrow} N$ and $N \rightarrow N / T \stackrel{g_{0}}{\rightarrow} P$. Now the composite factors as $M \rightarrow M / S \stackrel{f_{0}}{\rightarrow}$ $N \rightarrow N / T \stackrel{g_{0}}{\rightarrow} P$. Notice by construction that $f_{0}$ is an isomorphism, so Lemma 4.3 yields that $M /\left(S \cup f_{0}^{-1} T\right) \stackrel{\left.f_{0}\right|_{E_{M} \backslash\left(S \cup f_{0}^{-1} T\right)}}{\longrightarrow} N / T$ is an isomorphism. In particular we have a factorization $M \rightarrow M /\left(S \cup f^{-1} T\right) \stackrel{\left.f_{0}\right|_{E_{M} \backslash\left(S \cup f_{0}^{-1} T\right)} ^{\longrightarrow}}{\longrightarrow} N / T \stackrel{g_{0}}{\longrightarrow} P$; the latter two arrows in this

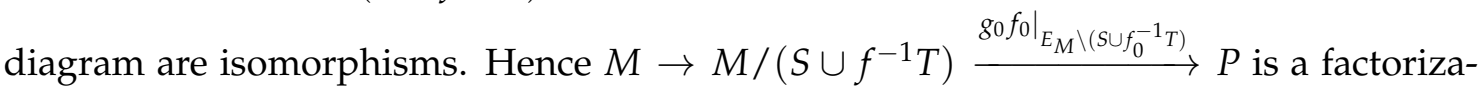
tion of $g f$ which shows this is an admissible epimorphism.

To see Properties 3, 4, and 5 we will appeal frequently to the following Lemma:

Lemma 4.9. For all $T \subseteq S \subseteq E_{M}$ with $*_{M} \in S \backslash T$, the following is a biCartesian square in $\mathcal{E}$ :

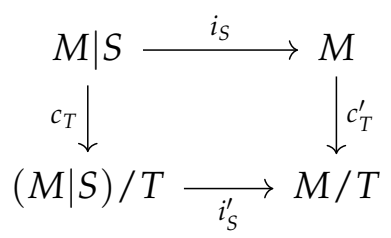

Proof. Notice trivially that the above square commutes.

Cartesian: Suppose that we have the following commutative diagram in $\mathcal{E}$ :

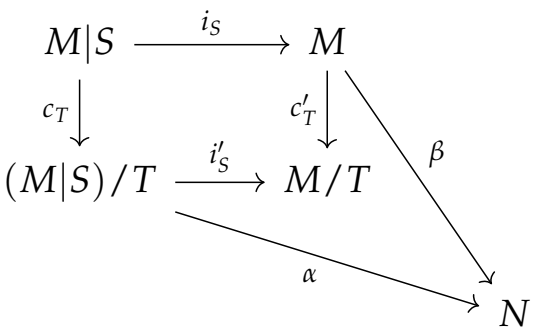

Define $\gamma=\left.\beta\right|_{E_{M} \backslash T}: M / T \rightarrow N$ and notice $\beta=\gamma c_{T}^{\prime}$ by construction and the fact that $\beta i_{S}=\alpha c_{T}$. Furthermore we have

$$
\alpha c_{T}=\beta i_{S}=\gamma c_{T}^{\prime} i_{S}=\gamma i_{S}^{\prime} c_{T}
$$

which yields $\alpha=\gamma i_{S}^{\prime}$ after noting trivially that $\left.c_{T}\right|_{S \backslash T}=\operatorname{id}_{S \backslash T}$.

Let $F \in \mathcal{F}(N)$ be arbitrary. By assumption we have that $\beta^{-1} F \in \mathcal{F}(M)$ and $\alpha^{-1} F \in$ $\mathcal{F}((M \mid S) / T)$. Now there is an $A \in \mathcal{F}(M)$ such that $T \subseteq A$ and $\alpha^{-1} F=(A \backslash T) \cap S$. On the other hand $\beta i_{S}=\alpha c_{T}$ yields the following:

$$
S \cap \beta^{-1} F=i_{S}^{-1} \beta^{-1} F=c_{T}^{-1} \alpha^{-1} F=T \cup \alpha^{-1} F=T \cup((A \backslash T) \cap S)
$$

Thus $T \subseteq S \cap \beta^{-1} F$ yields $T \subseteq \beta^{-1} F$, and so $\gamma^{-1} F=\left(\beta^{-1} F\right) \backslash T \in \mathcal{F}(M / T)$. Hence $\gamma$ is a strong map. Applying the forgetful functor $\mathbb{F}: \mathcal{E} \rightarrow \mathcal{S e t}_{\bullet}$, note that $\mathbb{F} \gamma$ is the 
pushout morphism of the corresponding square; $\gamma$ is thus uniquely determined in $\mathcal{E}$ by uniqueness of the underlying set map $\mathbb{F} \gamma$. Hence the square is Cartesian.

CoCartesian: Suppose that we have the following commutative diagram in $\mathcal{E}$ :

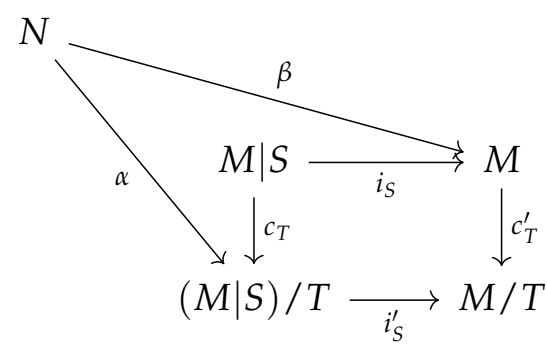

One observes that $\beta\left(E_{N}\right) \subseteq S$. Indeed, assume to the contrary that there is an $x \in E_{N} \backslash$ $\beta^{-1} S$; thus $\beta(x) \in E_{M} \backslash S$ and we have $\beta(x)=c_{T}^{\prime} \beta(x)$ as $T \subseteq S$. Now this yields $\beta(x)=i_{S}^{\prime} \alpha(x)=\alpha(x) \in S$, contradicting our initial assumption. Hence $\beta\left(E_{N}\right) \subseteq S$.

Define $\gamma: E_{N} \rightarrow S: x \mapsto \beta(x)$ and note that $\gamma$ is well-defined by our above argument. By construction $i_{S} \gamma=\beta$, and $i_{S}^{\prime} \alpha=c_{T}^{\prime} \beta=c_{T}^{\prime} i_{S} \gamma=i_{S}^{\prime} c_{T} \gamma$; hence we have $\alpha=c_{T} \gamma$ by injectivity of $i_{S}^{\prime}$.

Let $F \in \mathcal{F}(M)$ be arbitrary. Now $\gamma^{-1}(F \cap S)=\beta^{-1}(F \cap S)=\beta^{-1} F \in \mathcal{F}(N)$ as $\beta$ is a strong morphism. Hence $\gamma$ is a strong morphism. Applying the forgetful functor $\mathbb{F}: \mathcal{E} \rightarrow \mathcal{S e t}_{\bullet}$, note that $\mathbb{F} \gamma$ is the pullback morphism of this square; $\gamma$ is thus uniquely determined in $\mathcal{E}$. Hence the square is coCartesian.

We can now complete the proof that $\mathcal{E}$ is proto-exact.

Proposition 4.10 (Verifying Property 4). Every diagram $P \stackrel{i^{\prime}}{\longrightarrow} Q \stackrel{j^{\prime}}{\longleftarrow} N$ in $\mathcal{E}$ with $i^{\prime} \in$ $\mathfrak{M}$ and $j^{\prime} \in \mathfrak{E}$ can be completed to a biCartesian square

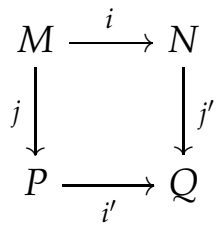

for some $M \in \mathcal{E}, i \in \mathfrak{M}, j \in \mathfrak{E}$.

Proof. Since $i^{\prime} \in \mathfrak{M}$, there exists $S \subseteq E_{Q}$ such that $i^{\prime}=i_{s} g$, where $g: P \rightarrow Q \mid S$ is an isomorphism and $i_{S}: Q \mid S \rightarrow Q$ (as in Lemma 4.6). Similarly, since $j^{\prime} \in \mathfrak{E}$, there exists $T \subseteq E_{N}$ such that $j^{\prime}=f c_{T}^{\prime}$, where $f: N / T \rightarrow Q$ is an isomorphism and $c_{T}^{\prime}: N \rightarrow N / T$ (as in Lemma 4.6). We prove that $M=N \mid\left(j^{\prime}\right)^{-1}(S)$ gives us the desired biCartesian square along with a canonical choice of $i$ and $j$. 
Let $A:=\left(j^{\prime}\right)^{-1}(S)$. Then one can easily see that $T \subseteq A, *_{N} \in A \backslash T$ and hence, from the above factorizations of $i^{\prime}$ and $j^{\prime}$, we obtain the following commuting diagram:

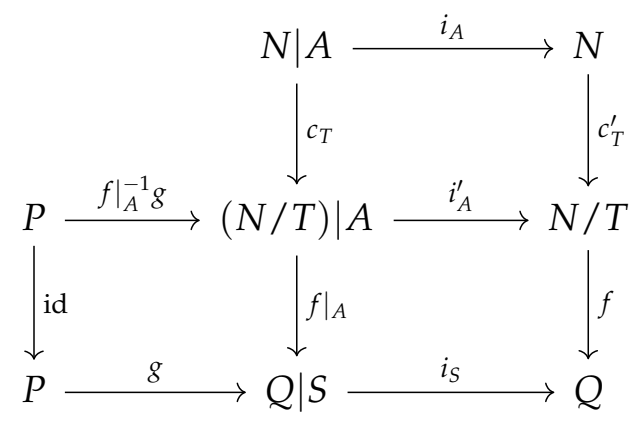

Now note that the following square is a commuting square in $\mathcal{E}$, where $i=i_{A}$ and $j=\left.g^{-1} f\right|_{A} c_{T}$ :

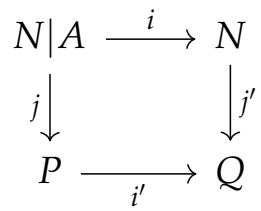

Then we have a factorization of $i$ as follows:

$$
N|A \stackrel{\text { id }}{\rightarrow} N| A \stackrel{i_{A}}{\rightarrow} N
$$

Using Lemma 4.4, we have a factorization of $j$ :

$$
N \mid A \stackrel{c_{T}}{\longrightarrow}(N \mid A) / T \stackrel{\left.g^{-1} f\right|_{A}}{\longrightarrow} P .
$$

Moreover $\left.g^{-1} f\right|_{A}$ is an isomorphism by Lemma 4.3. Hence $i \in \mathfrak{M}$ and $j \in \mathfrak{E}$.

To see that this square is Cartesian, note that every commuting diagram

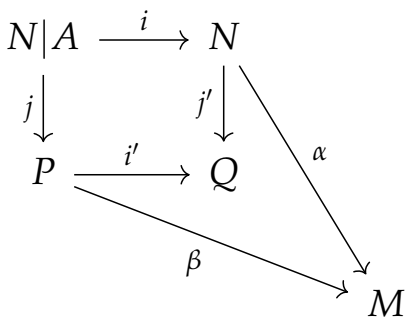

determines a corresponding commuting diagram

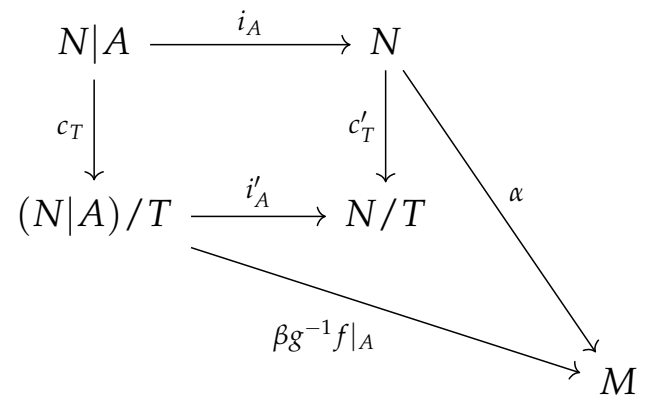


which admits a unique map $\delta: N / T \rightarrow M$ such that the diagram commutes by Lemma 4.9. On the other hand, this implies that $\gamma=\delta f^{-1}$ is the pushout of the original square by uniqueness of the pushout in $\mathcal{S e t}$.

To see that this square is coCartesian, note that every commuting diagram

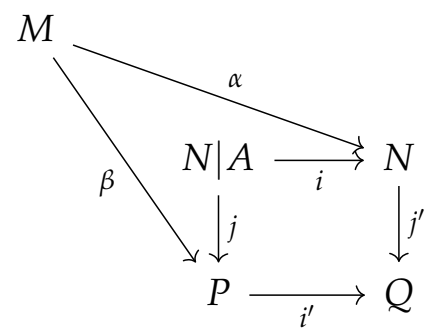

determines a corresponding commuting diagram

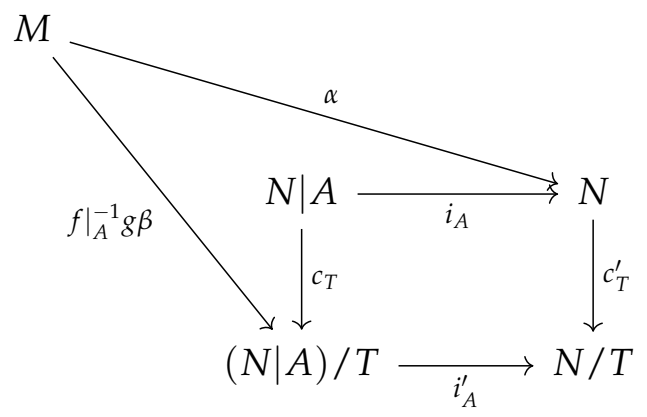

which admits a unique map $\gamma: M \rightarrow N \mid A$ such that the diagram commutes by Lemma 4.9. On the other hand, this implies that $\gamma$ is the pullback of the original square by uniqueness of the pullback in $\mathcal{S e t}_{\text {. }}$.

In particular, we have shown that every diagram $P \stackrel{i^{\prime}}{\hookrightarrow} Q \stackrel{j^{\prime}}{\leftarrow} N$ with arrows $i^{\prime} \in \mathfrak{M}$ and $j^{\prime} \in \mathfrak{E}$ determines a biCartesian square with new arrows $P \stackrel{j}{\leftarrow} M \stackrel{j}{\hookrightarrow} N$ where which $i \in \mathfrak{M}$ and $j \in \mathfrak{E}$.

Proposition 4.11 (Verifying Property 5). Every diagram $P \stackrel{j}{\longleftarrow} M \stackrel{i}{\longleftrightarrow} N$ in $\mathcal{E}$ with $i \in$ $\mathfrak{M}$ and $j \in \mathfrak{E}$ can be completed to a biCartesian square

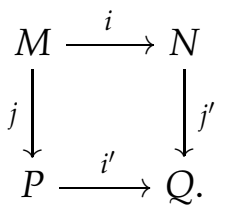

for some $Q \in \mathcal{E}, i^{\prime} \in \mathfrak{M}, j^{\prime} \in \mathfrak{E}$. 
Proof. Since $i \in \mathfrak{M}$ and $j \in \mathfrak{E}$, we have factorizations of $i$ and $j$ from which we obtain the following commuting diagram:

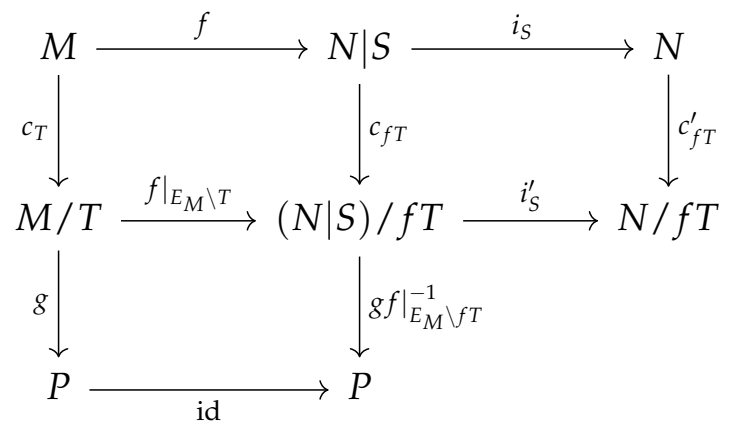

Now note that the following square is a commuting square in $\mathcal{E}$, where $i^{\prime}=\left.i_{S}^{\prime} f\right|_{E_{M} \backslash f T} g^{-1}$ and $j^{\prime}=c_{f T}^{\prime}$ :

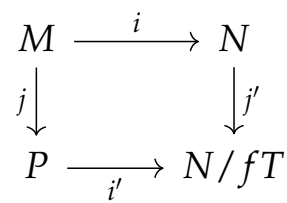

We have factorizations $N \stackrel{c_{f T}^{\prime}}{\longrightarrow} N / f T \stackrel{\text { id }}{\rightarrow} N / f T$ and $P \stackrel{\left.f\right|_{E_{M} \backslash f T} g^{-1}}{\longrightarrow}(N \mid S) / f T \stackrel{i_{S}^{\prime}}{\longrightarrow} N / f T$. Moreover $\left.f\right|_{E_{M} \backslash f T} g^{-1}$ is an isomorphism by Lemma 4.3. Hence $i^{\prime} \in \mathfrak{M}$ and $j^{\prime} \in \mathfrak{E}$.

To see that this square is Cartesian, note that every commuting diagram

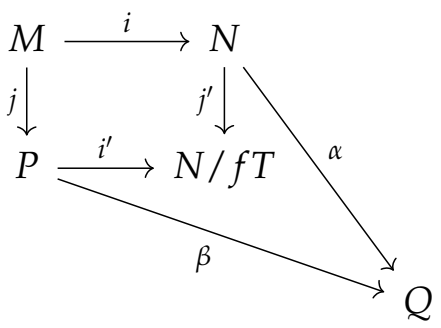

determines a corresponding commuting diagram

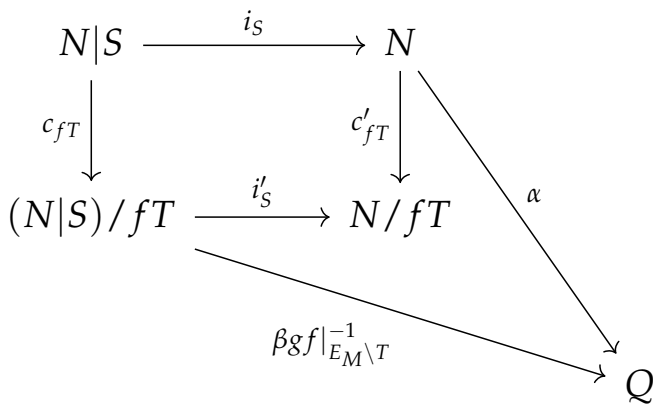

which admits a unique map $\gamma: N / T \rightarrow Q$ such that the diagram commutes by Lemma 4.9. On the other hand, this implies that $\gamma$ is the pushout of the original square by uniqueness of the pushout in $\mathcal{S e t}$. 
To see that this square is coCartesian, note that every commuting diagram

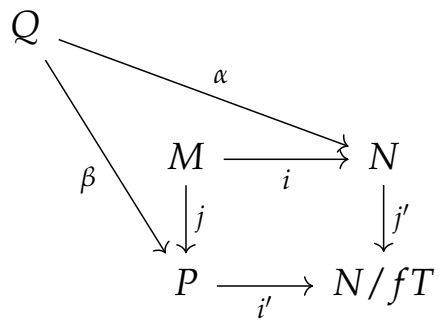

determines a corresponding commuting diagram

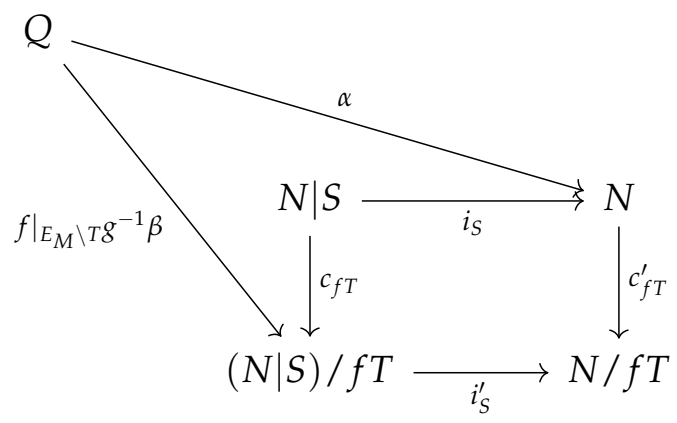

which admits a unique map $\delta: Q \rightarrow N \mid S$ such that the diagram commutes by Lemma 4.9. On the other hand, this implies that $\gamma=f^{-1} \delta$ is the pullback of the original square by uniqueness of the pullback in $\mathcal{S e t}$.

Proposition 4.12 (Verifying Property 3). A commuting square in $\mathcal{E}$ with $i, i^{\prime} \in \mathfrak{M}$ and $j, j^{\prime} \in$ E:

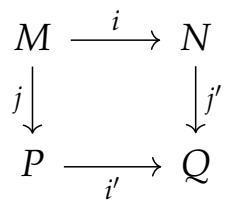

is Cartesian if and only if it is coCartersian.

Proof. Suppose the above square is either Cartesian or coCartesian. By the previous propositions, both $P \stackrel{j}{\leftarrow} M \stackrel{i}{\hookrightarrow} N$ and $P \stackrel{i^{\prime}}{\hookrightarrow} Q \stackrel{j^{\prime}}{\leftarrow} N$ can be completed to biCartesian squares in $\mathcal{E}$ having all arrows from $\mathfrak{M}$ and $\mathfrak{E}$. On the other hand, pullback and pushout objects are unique up to isomorphism. Thus the original square is necessarily biCartesian. Hence Cartesian and coCartesian are equivalent for all such squares.

Propositions 4.7, 4.8, 4.12, 4.10, and 4.11 thus complete our first proof of Theorem A.

\section{Mat. AS A PROTO-EXACT CATEGORY Via B-MODUleS}

In this section, we present another proof showing that Mat. is a proto-exact category by appealing to the recent work [3] of C. Crowley, N. Giansiracusa, and J. Mundinger. Our motivation of introducing the second proof is to shed some light on generalizing the 
current work to the case of Hopf algebras for matroids over hyperfields introduced by the authors of the current paper in [7].

5.1 Matroids as $\mathbb{B}$-modules. In [3] the authors give a very useful characterization of matroids and strong maps in terms of $\mathbb{B}$-modules, where $\mathbb{B}$ denotes the Boolean semifield $\mathbb{B}=\{0,1\}$ with

$$
1 \cdot 1=1,0 \cdot 1=1 \cdot 0=0 \cdot 0=0 \quad 0+0=0,0+1=1+0=1+1=1 .
$$

We proceed to review their construction. For a finite set $E$, we denote by $\mathbb{B}^{E}$ the free $\mathbb{B}$ module on $E$, with standard basis $\left\{e_{i}\right\}, i \in E$. The linear dual $\operatorname{Hom}_{\mathbb{B}}\left(\mathbb{B}^{E}, \mathbb{B}\right)$ is denoted $\left(\mathbb{B}^{E}\right)^{\vee}$, and has dual basis $\left\{x_{i}\right\}, i \in E$, with $\left\langle e_{i}, x_{j}\right\rangle=\delta_{i j}$.

Remark 5.1. In fact, the notion of free $\mathbb{B}$-modules is rather subtle. For instance, any 'free $\mathbb{B}$-module' of dimension $n$ does not have to be isomorphic to $\mathbb{B}^{n}$. See, $[19, \S 2]$ for details. Nonetheless, in this paper, we restrict ourselves to the case of free $\mathbb{B}$-modules of the form $\mathbb{B}^{E}$.

From now one, all matrids are assumed to be pointed. Given a matroid $M$, let $L_{M} \subseteq$ $\mathbb{B}^{E_{M}}$ be the $\mathbb{B}$-submodule generated by the support vectors of the cocircuits of $M$. They show:

Proposition 5.2 ([3]). Let $M$ be a matroid. Then $M$ is completely determined by the $\mathbb{B}$-module $L_{M}$ together with its embedding $L_{M} \hookrightarrow \mathbb{B}^{E_{M}}$.

For matroids $N, M$, a map $f: E_{N} \rightarrow E_{M}$, induces a $\mathbb{B}$-module map

$$
\begin{gathered}
f_{*}:\left(\mathbb{B}^{E_{N}}\right)^{\vee} \rightarrow\left(\mathbb{B}^{E_{M}}\right)^{\vee} \\
f_{*}\left(x_{i}\right)=x_{f(i)} .
\end{gathered}
$$

Taking the transpose (dual) we obtain a $\mathbb{B}$-module map

$$
f_{*}^{\vee}: \mathbb{B}^{E_{M}} \rightarrow \mathbb{B}^{E_{N}}
$$

Proposition 5.3 ([3]). $f: E_{N} \rightarrow E_{M}$ defines a strong map if and only if $f_{*}^{\vee}\left(L_{M}\right) \subseteq L_{N}$.

Definition 5.4. Let $\mathbb{B} \bmod _{E m b}$ denote the category with:

- Objects are embedded sub-modules $L \subseteq \mathbb{B}^{E}$ for a finite set $E$.

- Morphisms from $L \subseteq \mathbb{B}^{E}$ to $K \subseteq \mathbb{B}^{F}$ are commutative squares

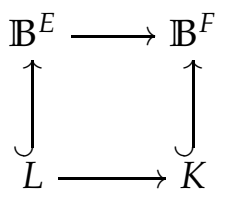

Then, one has the following: 
Proposition $5.5([3])$. There exists a faithful functor

$$
\mathcal{L}:\left(\text { Mat. }_{\bullet}\right)^{o p} \rightarrow \mathbb{B} m o d_{E m b}
$$

which assigns to a matroid $M$ the embedded sub-module $L_{M} \subseteq \mathbb{B}^{E_{M}}$ and to a strong map of matroids $f: N \rightarrow M$ the induced map (10).

The functor $\mathcal{L}$ is not full, and as the authors point out in [3], a general morphism in $\mathbb{B} \bmod _{E m b}$ between two objects in the essential image of $\mathcal{L}$ may be viewed as a "multivalued" strong map. One also obtains the following pleasant characterization of the restriction and contraction operations:

Proposition 5.6 ([3]). Let $M$ be a matroid, and $S \subseteq E_{M}$.

$$
\mathcal{L}(M \mid S)=\pi_{S}\left(L_{M}\right) \subseteq \mathbb{B}^{S}
$$

where

$$
\pi_{S}: \mathbb{B}^{E_{M}} \rightarrow \mathbb{B}^{S}
$$

is the canonical projection. Under the functor $\mathcal{L}$, the commutative square

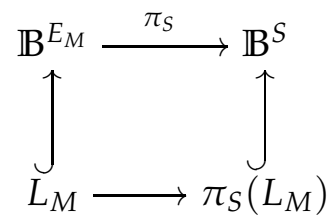

corresponds to the canonical strong inclusion $M \hookleftarrow M \mid S$

(2)

$$
\mathcal{L}(M / S)=L_{M} \cap \mathbb{B}^{E_{M} \backslash S} \subseteq \mathbb{B}^{E_{M} \backslash S},
$$

where $\mathbb{B}^{E_{M} \backslash S}$ denotes the $\mathbb{B}$-submodule of $\mathbb{B}^{E_{M}}$ consiting of vectors having 0 in all components corresponding to $S$. Under the functor $\mathcal{L}$, the commutative square

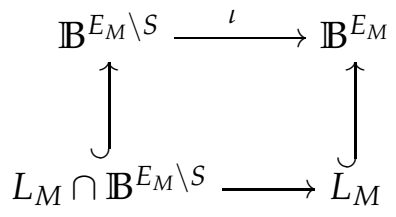

corresponds to the canonical strong map $M \rightarrow M / S$.

Theorem 5.7. Mat. has the structure of a proto-exact category

Proof. Mat. is pointed, with $(\{*\}, *)$ the zero object. We note that $\mathcal{L}((\{*\}, *))=0$ - the trivial $\mathbb{B}$-module. This verifies property (1) of Definition 2.1. The classes $\mathfrak{M}$, $\mathfrak{E}$ obviously contain all isomorphisms and are closed under composition, showing (2) of Definition 
2.1. To show the existence of the push-outs and pull-backs (4), (5), it suffices to work in the category $\mathbb{B} \bmod _{E m b}$ via the embedding $\mathcal{L}$, keeping in mind that it is contravariant.

Consider a diagram in Mat. of the kind considered in (4). Applying $\mathcal{L}$, this becomes a diagram in $\mathbb{B} \bmod _{E m b}$ of the form

$$
\pi\left(L_{M} \cap \mathbb{B}^{E_{M} \backslash T}\right) \subseteq \mathbb{B}^{E_{M} \backslash(T \cup S)} \underset{\ll}{\pi} L_{M} \cap \mathbb{B}^{E_{M} \backslash T} \subseteq \mathbb{B}^{E_{M} \backslash T \hookrightarrow} \stackrel{i}{\longrightarrow} L_{M} \subseteq B^{E_{M}}
$$

where $S, T \subseteq E_{M}$ are disjoint, and $\pi$ denotes the projection induced by the inclusion of sets $E_{M} \backslash(S \cup T) \subseteq E_{M} \backslash T$. The pushout of this diagram in $\mathbb{B} m o d_{E m b}$ is easily seen to be

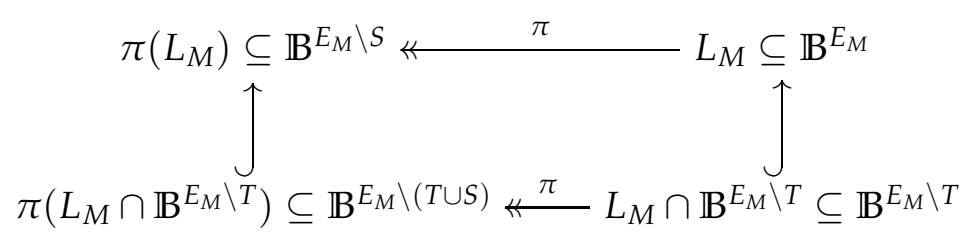

For a matroid $M, \pi\left(L_{M}\right) \subseteq \mathbb{B}^{E_{M} \backslash S}$ is isomorphic to $\mathcal{L}(M \mid S)$, which shows that the pullback of the diagram (4) exists in Mat., and that the completing maps lie in $\mathfrak{M}, \mathfrak{E}$ as desired.

Similarly, a diagram in Mat. of the kind (5), becomes after applying $\mathcal{L}$ a diagram in $\mathbb{B} m o d_{E m b}$ of the form

$$
\pi\left(L_{M}\right) \cap \mathbb{B}^{E_{M} \backslash(T \cup S)} \subseteq \mathbb{B}^{E_{M} \backslash(T \cup S)} \leftrightharpoons \pi\left(L_{M}\right) \subseteq \mathbb{B}^{E_{M} \backslash S} \underset{ }{\longleftarrow} L_{M} \subseteq \mathbb{B}^{E_{M}}
$$

for disjoint $S, T \subseteq E_{M}$. The pullback of this diagram in $\mathbb{B} m o d_{E m b}$ is

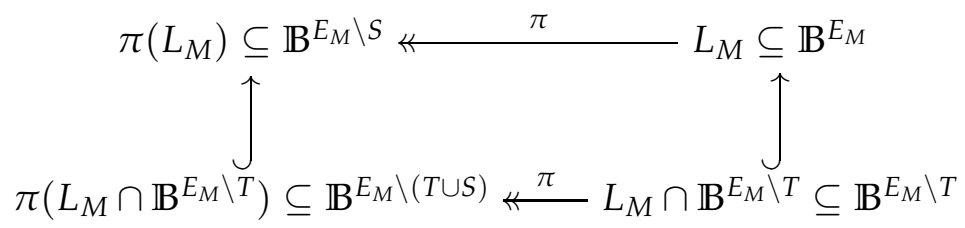

This shows that the pushout of the diagram (5) exists in Mat. Furthermore, comparing (4), (5) shows that property (3) holds. This completes the proof.

Remark 5.8. We note the following:

(1) The admissible sub-objects and quotient objects of $M \in$ Mat. correspond respectively to matroids $M \mid S$ and $M / S$ for subsets $S \subseteq \tilde{E}_{M}$.

(2) The indecomposable objects of Mat. are precisely the connected pointed matroids.

(3) The admissible sub-quotients of $M \in$ Mat. are precisely the pointed minors of $M$.

(4) The forgetful functor $\mathbb{F}:$ Mat. $\mapsto \mathcal{S}_{\bullet}$. is an exact functor of proto-exact categories. 
The above proof shows that the biCartesian completions of the diagrams from Definition (2.1) in Mat. are minors of the matroids in the diagrams. Let $\mathcal{M}$ be a collection of pointed matroids which is closed under taking pointed minors, and let $\operatorname{Mat}_{\mathbf{0}}(\mathcal{M})$ denote the full sub-category of Mat. generated by objects in $\mathcal{M}$. We then obtain

Theorem 5.9. Mat. $(\mathcal{M})$ has the structure of proto-exact category. It is a full sub-category of Mat..

\section{AlgEBRAIC K-THEORY OF MATROIDS}

6.1 K-theory of proto-exact categories. We begin by recalling the construction of the algebraic K-theory of a proto-exact category following $[6,11]$. Let $\mathcal{C}$ be a proto-exact category and let $\mathcal{S}_{n}=\mathcal{S}_{n}(\mathcal{C})$ denote the maximal groupoid in the category of diagrams of the form
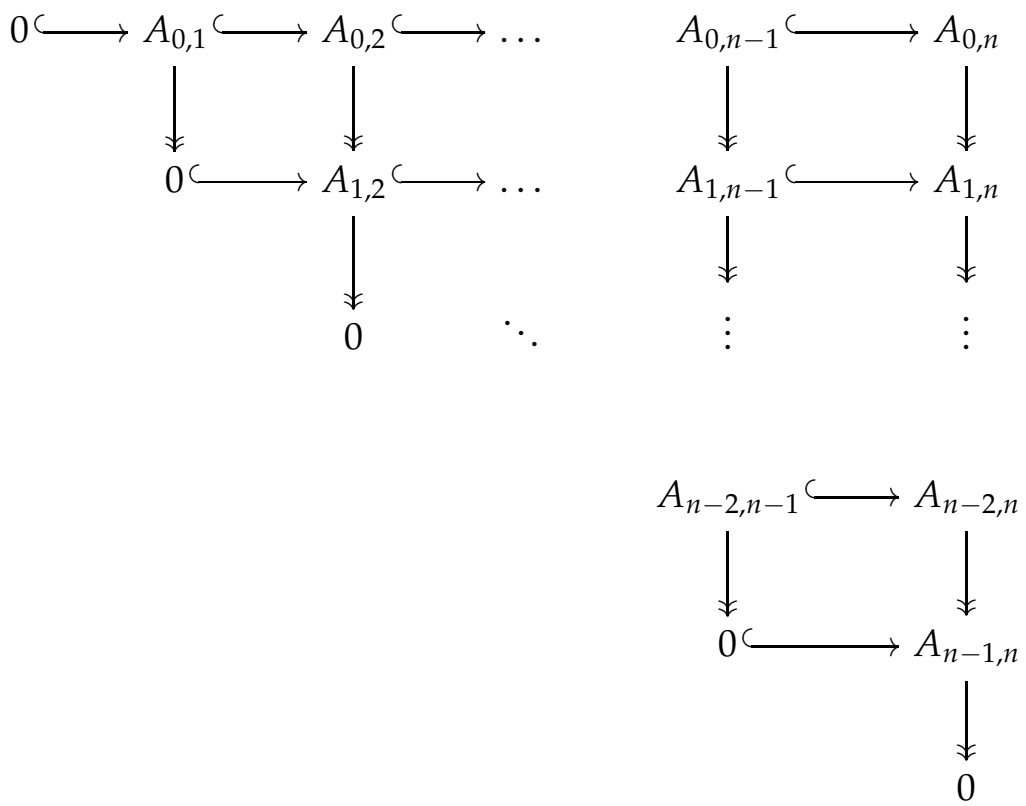

where all horizontal maps are in $\mathfrak{M}$ and all vertical maps in $\mathfrak{E}$, and all squares are required to be biCartesian. For every $0 \leq k \leq n$, there is a functor

$$
\partial_{k}: \mathcal{S}_{n} \rightarrow \mathcal{S}_{n-1}
$$

obtained by omitting in the diagram (11) the objects in the $k$ th row and $k$ th column and forming the composite of the remaining morphisms. Similarly, for every $0 \leq k \leq n$, there is a functor

$$
\sigma_{k}: \mathcal{S}_{n} \rightarrow \mathcal{S}_{n+1}
$$

given by replacing the $k$ th row by two rows connected via identity maps and replacing the $k$ th column by two columns connected via identity maps. $\mathcal{S}_{*}(\mathcal{C})$ together with the $\partial_{*}, \sigma_{*}$ forms a simplicial object in the category of groupoids. 
Definition 6.1. The K-theory of $\mathcal{C}$ is defined by

$$
K_{n}(\mathcal{C})=\pi_{n+1}\left|\mathcal{S}_{\bullet} \mathcal{C}\right|,
$$

where $\left|\mathcal{S}_{\bullet} \mathcal{C}\right|$ denotes the geometric realization of $\mathcal{S}_{\bullet} \mathcal{C}$.

Remark 6.2. One may also develop K-theory of proto-exact categories via a version of Quillen's Q-construction. It is shown in [11] that this approach leads to isomorphic Kgroups. In particular, Theorem 1.1, whose original proof used the Q-construction, remains valid if the K-theory of $\mathcal{S e t}_{\bullet}$ is defined as in Definition 6.1.

The Grothendieck group $K_{0}(\mathcal{C})$ can be described explicitly as the free group on symbols $[A], A \in \operatorname{Iso}(\mathcal{C})$, modulo the relations $[B]=[A][C]$ for each admissible short exact sequence

$$
A \hookrightarrow B \rightarrow C .
$$

When $\mathcal{C}$ admits direct sums for which

$$
A \hookrightarrow A \oplus B \rightarrow B \text { and } B \hookrightarrow A \oplus B \rightarrow A
$$

are both admissible, which is the case for $\mathcal{C}=$ Mat., $K_{0}(\mathcal{C})$ is Abelian, and can be described as the free Abelian group on $[A] \in \operatorname{Iso}(\mathcal{C})$ modulo the relations $[B]=[A]+[C]$. $\mathrm{K}$-theory is functorial under exact functors, meaning that an exact functor between protoexact categories $F: \mathcal{C} \mapsto \mathcal{D}$ induces group homomorphisms

$$
F_{*}: K_{n}(\mathcal{C}) \mapsto K_{n}(\mathcal{D})
$$

compatible with composition.

6.2 K-theory of Mat.. We begin by calculating the Grothendieck group of Mat.. Given a non-zero pointed matroid $M$ and $e \in \tilde{E}_{M}$, we have an admissible short exact sequence

$$
M \mid e \hookrightarrow M \rightarrow M / e
$$

Iterating this procedure shows that the class of any $M \in$ Mat. can be expressed as a sum of pointed matroids with one-element ground sets. There are two non-isomorphic such matroids, denoted $\mathbf{a}, \mathbf{b}$, where $r k(\mathbf{a})=1$ and $r k(\mathbf{b})=0$ (i.e. $\mathbf{b}$ is a "non-zero pointed loop"). They span $K_{0}$ (Mat.) and are easily seen to be independent, since rank and ground set cardinality is additive in admissible short exact sequences. We have thus proved the following:

Theorem 6.3. There is an isomorphism

$$
K_{0}\left(\text { Mat. }_{\bullet}\right) \rightarrow \mathbb{Z} \oplus \mathbb{Z}
$$

determined by

$$
M \rightarrow\left(r k(M),\left|E_{M}\right|-r k(M)\right)
$$


for each pointed matroid $M$.

The forgetful functor $\mathbb{F}:$ Mat. $\rightarrow$ Set. sending a pointed matroid to its ground set has a left adjoint (see [12]) $G: \mathcal{S e t}_{\bullet} \rightarrow$ Mat॰ sending a pointed set $E$ to the "free pointed matroid on $E^{\prime \prime}$. More precisely, $G(E)$ is the pointed matroid whose flats consist of all subsets of $E$ containing the basepoint. We have $\mathbb{F} \circ \mathbb{G}=I$, which implies the following:

Theorem 6.4. There are injective group homomorphisms

$$
\pi_{n}^{s}(\mathrm{~S}) \simeq K_{n}\left(\mathcal{S e t}_{\bullet}\right) \hookrightarrow K_{n}\left(\text { Mat }_{\bullet}\right)
$$

for all $n \geq 0$.

This shows in particular that $K_{n}$ (Mat.) is in general non-trivial for $n>0$, and contains interesting information of a homotopy-theoretic nature.

\section{HMat. AND THE MATROID-MinOR HOPF ALGEBRA}

In this section, we relate the Hall algebra of $\mathcal{E}=$ Mat. ( and more generally of the categories Mat. $(\mathcal{M}))$ to the Matroid-Minor Hopf algebras introduced by W. R. Schmitt in [22]. We begin by reviewing the latter, adapting to the case of pointed matroids.

7.1 The Matroid-Minor Hopf algebra. Let $\mathcal{M}$ be a collection of pointed matroids which is closed under taking pointed minors and direct sums and $\mathcal{M}_{\text {iso }}$ be the set of isomorphism classes of pointed matroids in $\mathcal{M}$. Let $[M]$ be the isomorphism class of a pointed matroid $M$ in $\mathcal{M} . \mathcal{M}_{\text {iso }}$ is equipped with a natural commutative monoid structure, via the pointed direct sum, as follows:

$$
\left[M_{1}\right] \cdot\left[M_{2}\right]:=\left[M_{1} \oplus M_{2}\right]
$$

and the identity $[(\{*\}, *)]$, the equivalence class of the zero pointed matroid. Let $k\left[\mathcal{M}_{\text {iso }}\right]$ be the monoid algebra of $\mathcal{M}_{\text {iso }}$ over a field $k$.

In [22] Schmitt constructs a comultiplication and counit:

- (Coproduct)

$$
\Delta: k\left[\mathcal{M}_{\text {iso }}\right] \rightarrow k\left[\mathcal{M}_{\text {iso }}\right] \otimes_{k} k\left[\mathcal{M}_{\text {iso }}\right], \quad[M] \mapsto \sum_{S \subseteq \widetilde{E}_{M}}\left[\left.M\right|_{S}\right] \otimes[M / S] .
$$

- (Counit)

$$
\varepsilon: k\left[\mathcal{M}_{\text {iso }}\right] \rightarrow k, \quad[M] \mapsto \begin{cases}1 & \text { if } E_{M}=\varnothing \\ 0 & \text { if } E_{M} \neq \varnothing,\end{cases}
$$

$k\left[\mathcal{M}_{\text {iso }}\right]$ carries a natural grading, where $\operatorname{deg}\left(M, *_{M}\right)=\# \widetilde{E}_{M}$. With the above maps and grading, $k\left[\mathcal{M}_{\text {iso }}\right]$ becomes a graded connected bialgebra and hence, from the result 
of M. Takeuchi [27], $k\left[\mathcal{M}_{\text {iso }}\right]$ has a unique Hopf algebra structure with a unique antipode $S$ given by:

$$
S=\sum_{i \in \mathbb{N}}(-1)^{i} m^{i-1} \circ \pi^{\otimes i} \circ \Delta^{i-1}
$$

where $m^{-1}$ is a canonical injection from $k$ to $k\left[\mathcal{M}_{\text {iso }}\right], \Delta^{-1}:=\varepsilon$, and $\pi: k\left[\mathcal{M}_{\text {iso }}\right] \rightarrow k\left[\mathcal{M}_{\text {iso }}\right]$ is the projection map defined by

$$
\left.\pi\right|_{k\left[\mathcal{M}_{\text {iso }}\right]_{n}} \begin{cases}\text { id } & \text { if } n \geq 1 \\ 0 & \text { if } n=0\end{cases}
$$

and extended linearly to $k\left[\mathcal{M}_{\text {iso }}\right]$.

Remark 7.1. The requirement that $\mathcal{M}$ be closed under direct sums is only needed to define the algebra structure. The coalgebra structure requires only that $\mathcal{M}$ be closed under taking minors. If $\mathcal{M}_{1} \subseteq \mathcal{M}_{2}$, then $k\left[\mathcal{M}_{1}\right]$ is a Hopf subalgebra of $k\left[\mathcal{M}_{2}\right]$.

The dual Hopf algebra of $k\left[\mathcal{M}_{\text {iso }}\right]$, denoted $k\left[\mathcal{M}_{\text {iso }}\right]^{*}$, is described explicitly in $[1,16]$. It is shown that $k\left[\mathcal{M}_{\text {iso }}\right]^{*}$ is isomorphic to $\left\{f: \mathcal{M}_{\text {iso }} \rightarrow k\right\}$, with the product given by the convolution:

$$
f \diamond g([M])=\sum_{S \subseteq \widetilde{E}_{M}} f([M \mid S]) g([M / S])
$$

and coproduct

$$
\Delta(f)([M],[N]):=f([M \oplus N])
$$

Comparing this with the Hopf structure of the Hall algebra $\mathrm{H}_{\text {Mat. }(\mathcal{M})}$ of Section 2.1, we see that the coproducts agree, and the algebra structures are opposite of each other; i.e. $-\diamond^{o p}$. However, every enveloping algebra possesses an algebra anti-automorphism which fixes the coproduct. We thus obtain:

Theorem 7.2. Let $\mathcal{M}$ be a collection of pointed matroids closed under taking pointed minors and direct sums. Then $\mathrm{H}_{\text {Mat. } \mathcal{M}} \simeq k\left[\mathcal{M}_{\text {iso }}\right]^{*}$, where $k\left[\mathcal{M}_{\text {iso }}\right]^{*}$ denotes Schmitt's matroid-minor Hopf algebra attached to the collection $\mathcal{M}$. $\mathrm{H}_{\text {Mat. }} \mathcal{M} \simeq \mathbb{U}\left(\delta_{[M]}\right),[M] \in$ $\mathcal{M}_{\text {iso }}$ ind, where $\mathcal{M}_{\text {iso }}$ ind denotes the isomorphism classes of connected pointed matroids in $\mathcal{M}_{\text {iso }}$.

\section{REFERENCES}

[1] Henry Crapo and William Schmitt, A free subalgebra of the algebra of matroids, European J. Combin. 26 (2005), no. 7, 1066-1085.

[2] Chenghao Chu, Oliver Lorscheid, and Rekha Santhanam, Sheaves and K-theory for $\mathbb{F}_{1}$-schemes, Adv. Math. 229 (2012), no. 4, 2239-2286.

[3] C. Crowley, N. Giansiracusa, and J. Mundinger, A module-theoretic approach to matroids, preprint (2017), available at arXiv:1712.03440. 
[4] Anton Deitmar, Remarks on zeta functions and K-theory over $\mathbf{F}_{1}$, Proc. Japan Acad. Ser. A Math. Sci. 82 (2006), no. 8, 141-146.

[5] Tobias Dyckerhoff, Higher categorical aspects of Hall algebras, preprint, available at arXiv: 1505.06940.

[6] Tobias Dyckerhoff and Mikhail Kapranov, Higher Segal Spaces I, preprint, available at arXiv: 1212. 3563.

[7] Chris Eppolito, Jaiung Jun, and Matt Szczesny, Hopf algebras for matroids over hyperfields, preprint, available at arXiv: 1712.08903.

[8] Imma Galvez, Joachim Kock, and Andrew Tonks, Decomposition spaces, incidence algebras and Möbius inversion I: basic theory, Adv. Math., to appear, available at arXiv:1512.07573.

[9] _ـ Decomposition spaces, incidence algebras and Möbius inversion II: completeness, length filtration, and finiteness, Adv. Math., to appear, available at arXiv:1512.07577.

[10] _ Decomposition spaces, incidence algebras and Möbius inversion III: the decomposition space of Möbius intervals, Adv. Math., to appear, available at arXiv:1512.07580.

[11] Jeroen Hekking, Segal Objects in Homotopical Categories $\mathcal{E}$ K-theory of Proto-exact Categories, Master's Thesis, Univ. of Utrecht, 2017, https://www. universiteitleiden.nl/binaries/content/assets/science/mi/scripties/master/hekking_master.pdf.

[12] Chris Heunen and Vaia Patta, The category of matroids, Applied Categorical Structures 26 (2018), no. 2, 205-237.

[13] Andrew Hubery, From Triangulated Categories to Lie Algebras: a theorem of Peng and Xiao, Trends in Representation Theory of Algebras and Related Topics, Contemp. Math. 406 (2006), 51-66.

[14] Mikhail Kapranov, Eisenstein series and quantum affine algebras, Journal of Mathematical Sciences 84 (1997), no. 5, 1311-1360.

[15] Mikhail Kapranov, Olivier Schiffmann, and Eric Vasserot, The Hall algebra of a curve, Selecta Math. (N.S.) 23 (2017), no. 1, 117-177.

[16] Woong Kook, Victor Reiner, and Dennis Stanton, A convolution formula for the Tutte polynomial, J. Combin. Theory Ser. B 76 (1999), no. 2, 297-300.

[17] Kobi Kremnizer and Matt Szczesny, Feynman graphs, rooted trees, and Ringel-Hall algebras, Comm. Math. Phys. 289 (2009), no. 2, 561-577.

[18] Jean-Louis Loday and María Ronco, Combinatorial Hopf algebras, Quanta of Maths 11 (2010), 347-383.

[19] Grigory Mikhalkin and Ilia Zharkov, Tropical curves, their Jacobians and theta functions, Curves and abelian varieties 465 (2008), 203-230.

[20] James Oxley, Matroid theory, Vol. 3, Oxford University Press, USA, 2006.

[21] Olivier Schiffmann, Lectures on Hall algebras, Geometric methods in representation theory. II, Sémin. Congr., 24-II, Soc. Math. France, Paris (2012), 1-141.

[22] William R Schmitt, Incidence Hopf algebras, J. Pure Appl. Algebra 96 (1994), no. 3, 299-330.

[23] Matt Szczesny, Incidence categories, J. Pure Appl. Algebra 215 (2011), no. 4, 303-309.

[24] _ On the Hall algebra of coherent sheaves on $\mathbb{P}^{1}$ over $\mathbb{F}_{1}$, J. Pure Appl. Algebra 216 (2012), no. 3, $662-672$.

[25] _ Representations of quivers over $\mathbb{F}_{1}$ and Hall algebras, Int. Math. Res. Not. 2012 (2012), no. 10, 23772404.

[26] _ On the Hall algebra of semigroup representations over $\mathbb{F}_{1}$, Math. Z. 276 (2014), no. 1-2, 371-386.

[27] Mitsuhiro Takeuchi, Free Hopf algebras generated by coalgebras, Journal of the Mathematical Society of Japan 23 (1971), no. 4, 561-582.

Department of Mathematical Sciences, Binghamton University, Binghamton, NY

eppolito@math.binghamton.edu 
Department of Mathematical Sciences, Binghamton University, Binghamton, NY jjun@math.binghamton.edu

Department of Mathematics and Statistics, Boston University, 111 Cumminton Mall, Boaston szczesny@math.bu.edu 